DOE/NV/11718--1280

DOE/NV/25946--024

\title{
Time-resolved hard x-ray spectrometer
}

\author{
Kenneth Moy ${ }^{\mathrm{a}}$, Ian McKenna ${ }^{\mathrm{a}}$, Thomas Keenan ${ }^{\mathrm{a}}$, Michael Cuneo ${ }^{\mathrm{b}}$ \\ ${ }^{a}$ NSTec / Special Technologies Laboratory, 5520 Ekwill St., Ste B, Santa Barbara, CA USA 93111 \\ ${ }^{\mathrm{b}}$ Sandia National Laboratories, New Mexico, PO Box 5800, Albuquerque, NM USA 87185
}

\begin{abstract}
Wired array studies are being conducted at the SNL Z accelerator to maximize the $\mathrm{x}$-ray generation for inertial confinement fusion targets and high energy density physics experiments. An integral component of these studies is the characterization of the time-resolved spectral content of the x-rays. Due to potential spatial anisotropy in the emitted radiation, it is also critical to diagnose the time-evolved spectral content in a space-resolved manner. To accomplish these two measurement goals, we developed an x-ray spectrometer using a set of high-speed detectors (silicon PIN diodes) with a collimated field-of-view that converged on a 1-cm-diameter spot at the pinch axis. Spectral discrimination is achieved by placing high $\mathrm{Z}$ absorbers in front of these detectors. We built two spectrometers to permit simultaneous different angular views of the emitted radiation. Spectral data have been acquired from recent $Z$ shots for the radial and polar views. UNSPEC ${ }^{1}$ has been adapted to analyze and unfold the measured data to reconstruct the $\mathrm{x}$-ray spectrum. The unfold operator code, $\mathrm{UFO}^{2}$, is being adapted for a more comprehensive spectral unfolding treatment.
\end{abstract}

Keywords: $x$-ray spectroscopy, instrumentation, spectral modeling, $Z$ accelerator

\section{INTRODUCTION}

The world-record-setting $\mathrm{x}$-ray power produced by the $\mathrm{Z}$ facility at Sandia National Laboratories using pinched tungsten wire arrays has led to forefront research in high-energy density physics that includes radiation transport, radiation coupling in materials at extreme conditions, and inertial confinement fusion. As a core diagnostic for $\mathrm{Z}$ shots, calibrated high-bandwidth, carbon-cathode x-ray diodes (XRD) are fielded with a judicious combination of filters to characterize the low end of the spectrum during the time evolution of the output flux. Since the emitted radiation is predominantly composed of low energy x-rays, this XRD data can be fit to a Planckian distribution to provide a measure of the x-ray power. However, to gain a better understanding of the pinch physics, it is necessary to characterize as well the high energy photons beyond $20 \mathrm{keV}$, generated by fast-electron bremsstrahlung in the pinch region. We extended the spectral characterization of the output flux by developing a time-resolved hard x-ray spectrometer (HXRS) based on differential absorption of emitted photons from the pinched source.

\section{HXRS DESCRIPTION}

The HXRS employs ultrafast-response-detectors (Emerge Semiconductor Si PIN diodes s/n 003-PIN-250: 3-mm² active area, $250 \mu \mathrm{m}$ depletion depth) arrayed behind a collimation assembly to shield hard x-rays up to a few MeV energy. The detectors are placed behind channels bored in the collimation assembly to view a coincident area of the radiation source a fixed distance away. Various absorber materials and thicknesses are placed inside these channels to pass the spectral content of interest. Each detector trace is recorded by a high-bandwidth real-time oscilloscope. The calibrated detector responses of Fig. 1 are combined with the measured data and absorber characteristics as input into an unfold algorithm to determine the spectral shape of the radiation source. The net result is that this instrument can measure the time-evolved emission history of a hard x-ray radiation source over a spectral region determined by the combined absorbers. The time resolution of a measurement is limited by the system bandwidth combination of detectors, electrical cabling, and data acquisition instruments, in quadrature. 
Ideally, the number of detectors used in the spectrometer is limited by space and cost of high-bandwidth real-time oscilloscope channels, but a minimum of six data and one background channels is needed to provide sufficient data for unfolding algorithms to yield a credible spectral shape.

\section{PD641 (003-PIN-250), -500V bias, IAC}

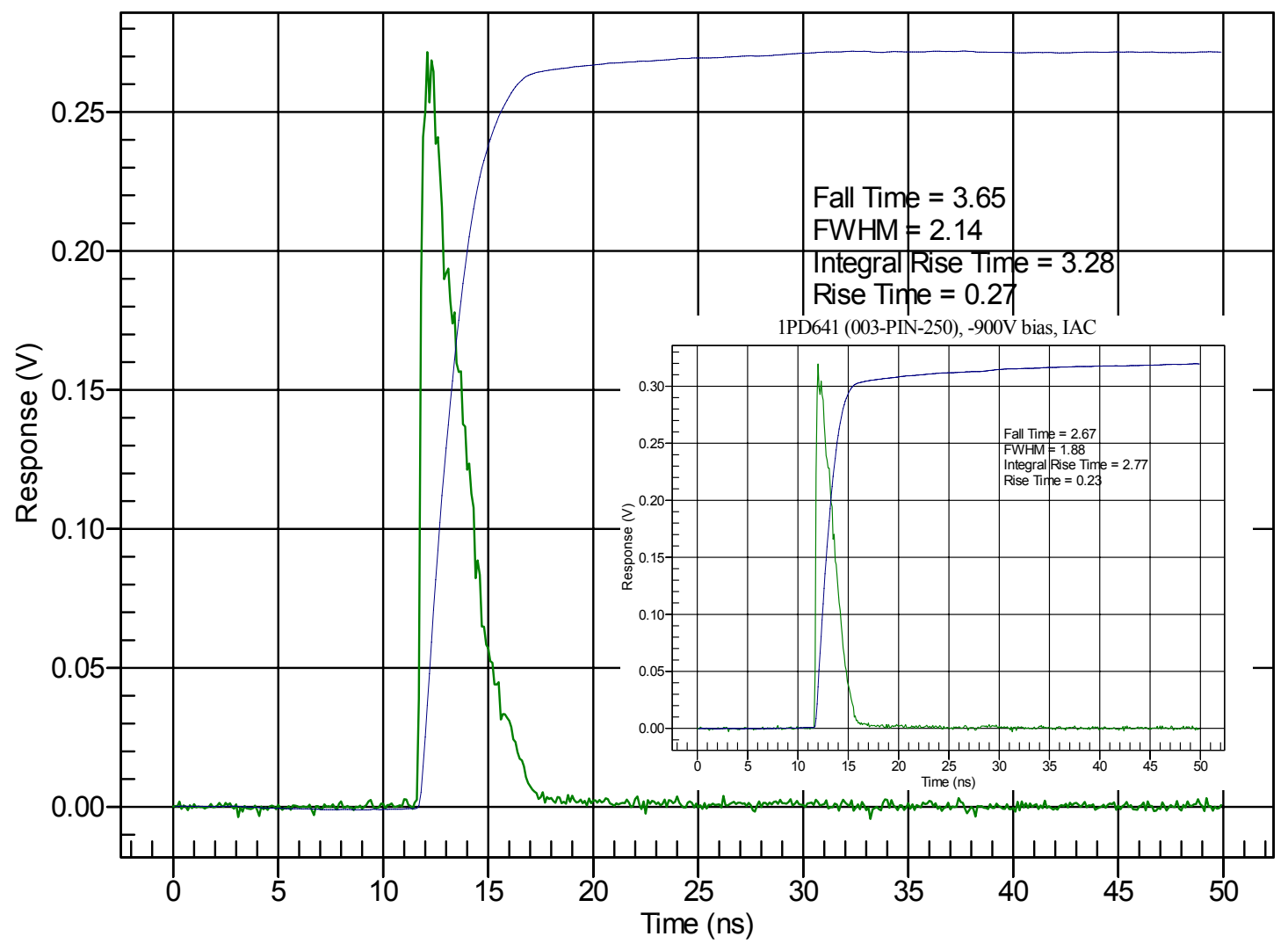

Fig. 1. Emerge Semiconductor Si PIN diode pulse shape at two reverse bias voltages: $-500 \mathrm{~V},-900 \mathrm{~V}$ (inset); as measured in a 16-MeV bremsstrahlung beam at the Idaho Accelerator Center electron linac.

Two versions of the HXRS were developed. The original prototype consists of seven detectors/channels, six for spectral measurements using absorber/pinholes and one blanked off to measure background (Fig. 2). The detectors and collimation assembly are fully housed inside a cylindrical pig filled with stainless steel balls for additional radiation shielding. The silicon PIN diodes (see Fig. 2) are mounted behind a 10.2-cm-long x 7.6-cm-diameter cylindrical tungsten collimation assembly to attenuate hard x-rays. Channels filled with 12.7 -mm-diameter x 2.5 -cm-long tungsten plugs precisely machined with 1-mm-diameter pinholes are used in this assembly to permit each detector to view a coincident 1-cm-diameter radiation source half a meter away (Fig. 3). Different collimation assemblies can be substituted to permit the same coincident view area at the source plane but at other source-to-HXRS distances. Absorber materials and thicknesses tailored to pass the spectral content of interest are placed in front of the pinholes between the source and detectors. The background channel is completely filled with solid tungsten plugs.

A second, more refined and more compact version consists of 13 detectors/channels, 12 for spectral measurements and 1 background (Fig. 4). Radiation and EMP shielding is achieved using square tungsten plates stacked together to surround the collimation assembly and detectors instead of the prototype cylindrical pig filled with stainless steel balls. With twelve channels, this intrument can be configured with suitable filter combinations employing the "Ross pair" technique ${ }^{3,4}$ to sample the spectral content directly below $\sim 115.6 \mathrm{keV}\left(\mathrm{U} \mathrm{K}_{\alpha}\right)$. 


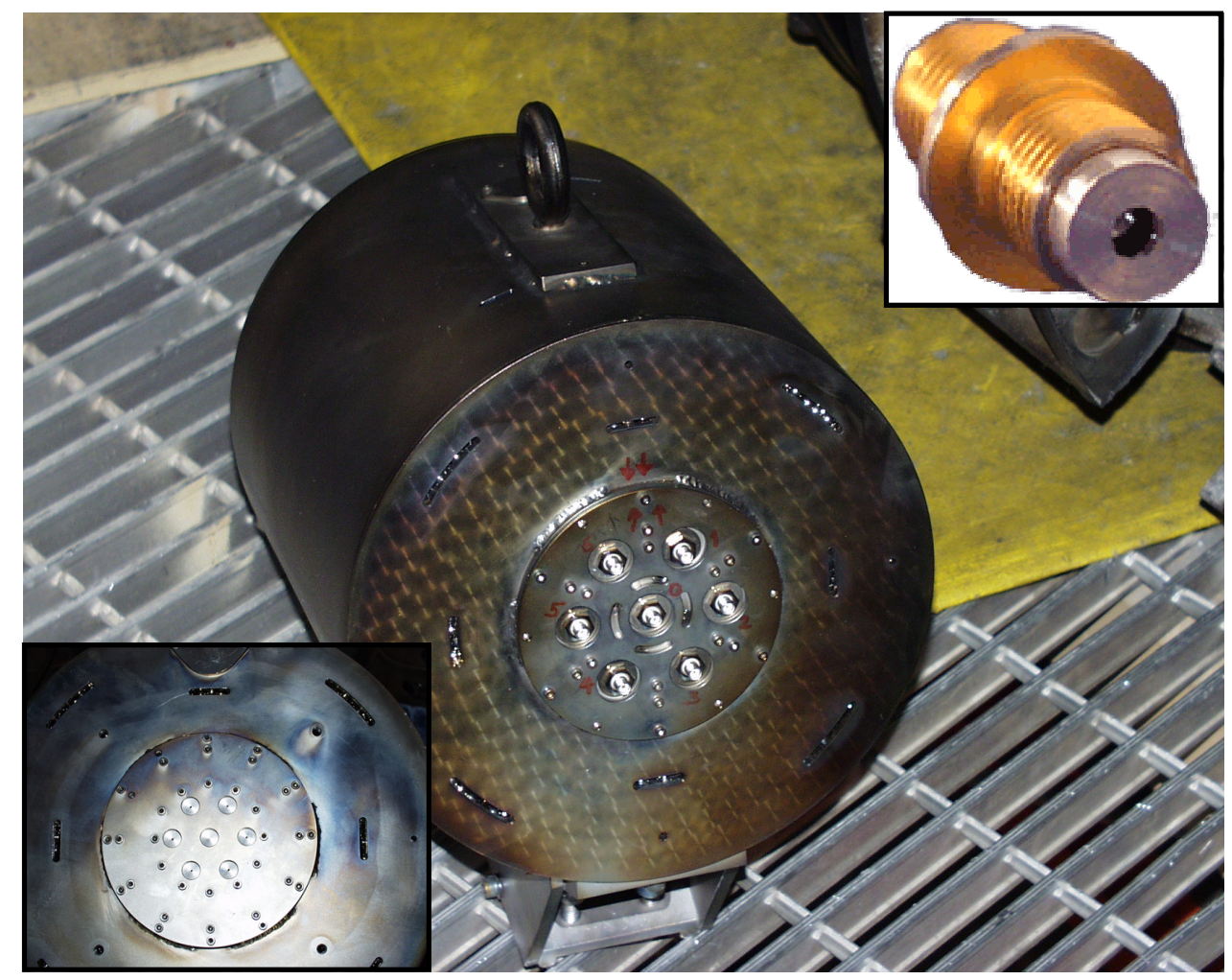

Fig. 2. Prototype six-channel spectrometer. Inset top-right: Emerge Semiconductor Si PIN diode. Inset bottom-left: front surface showing tungsten plugs with 1-mm diameter pinholes.

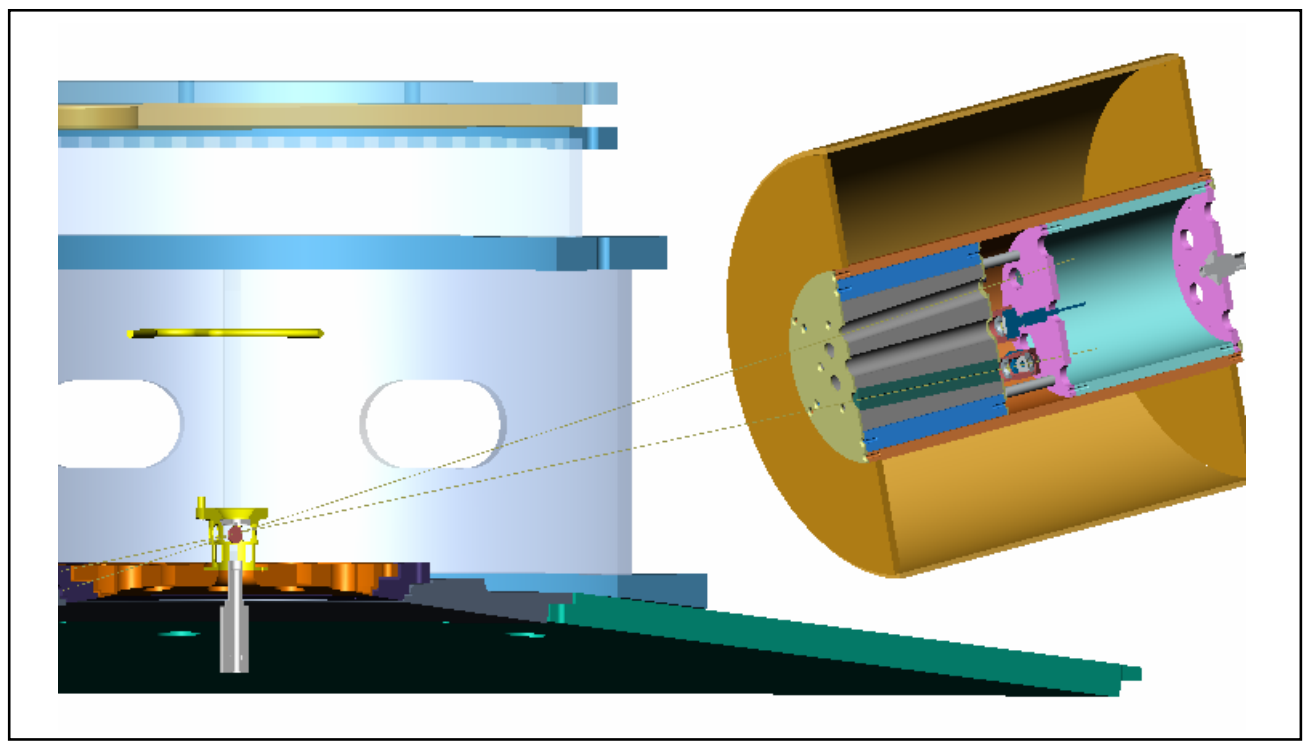

Fig. 3. Prototype seven-channel spectrometer field geometry. All seven detectors are viewing a coincident $1-\mathrm{cm}$-diameter area at a distance of $0.5 \mathrm{~m}$. 


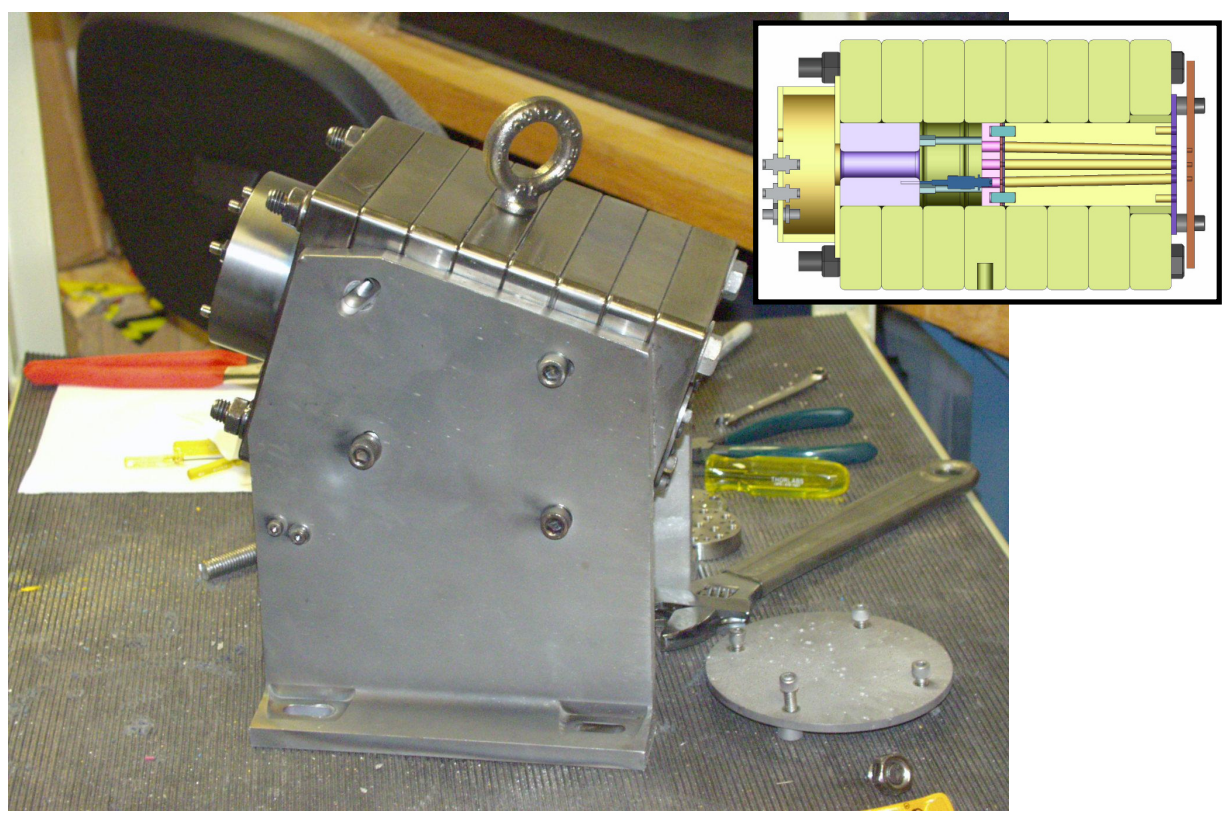

Fig. 4. Compact 13-channel spectrometer. Inset shows a rendered cross sectional slice of the interior.

Two compact spectrometers were built to view the pinch, one for taking a radial view at $50 \mathrm{~cm}$ and the other at an axial view from above at $107 \mathrm{~cm}$. Both instruments are identical except for the change in collimation assemblies in which a shallower angle in the collimation channels permits a longer object-to-detector view. Since the collimation length is the same, the axial field of view is $2-\mathrm{cm}$ diameter at the pinch plane. To assure an unobstructed view of the pinch, mounting fixtures are designed to raise and tilt the housing assembly above the MITL plates. As shown in Fig. 5, the radial fixture allows the field-of-view to pass directly through one of several view ports cut in the center section blast shield, while the axial mounting fixture attaches to a rack on top of the blast shield and allows the spectrometer to look down through port-holes cut into the blast shield lid. Class IIIA He-Ne penlight lasers are used to align the spectrometers. The lasers were permanently seated into modified plugs and aligned using a lathe to center the laser beam axis with the plug. After mounting, the spectrometer is finely aligned by replacing the plugs from several channels with the laser-seated plugs, and the housing is adjusted until the laser beam spots converge over the wire array. Prior to taking data, these laser-plugs are replaced with standard 1-mm-diameter plugs and absorbers tailored for the spectral bands of interest.

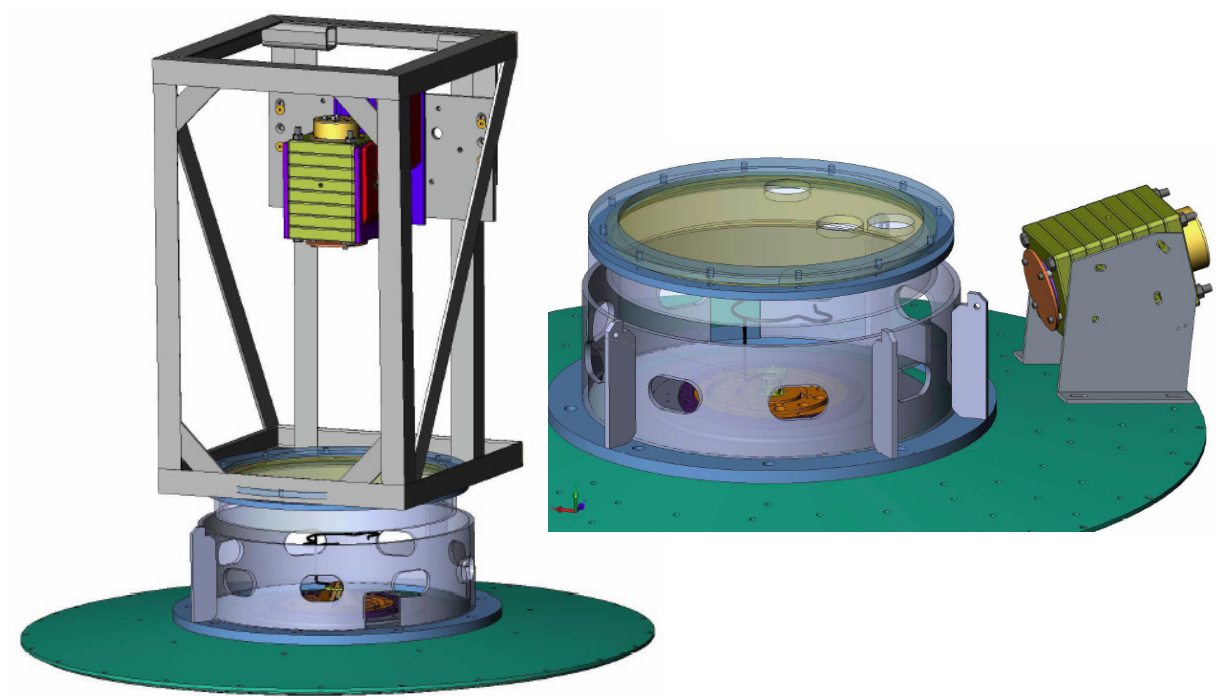

Fig. 5. Compact 12-channel spectrometer in axial (left) and radial (right) installation. 


\section{HARD X-RAY SPECTRUM}

\subsection{Model}

The seven-channel differential absorption spectrometer employs multiple channels to exploit high-pass filtering or spectral cut-on (Figs. 6 and 7) using metal absorbers. Detector spectral response (Fig. 8) is based on the fraction of photon energy absorbed by the $250-\mu \mathrm{m}$-thick silicon crystal. The electron-pairs generated in the active volume are collected on the reverse-biased electrodes to yield the pulse response.

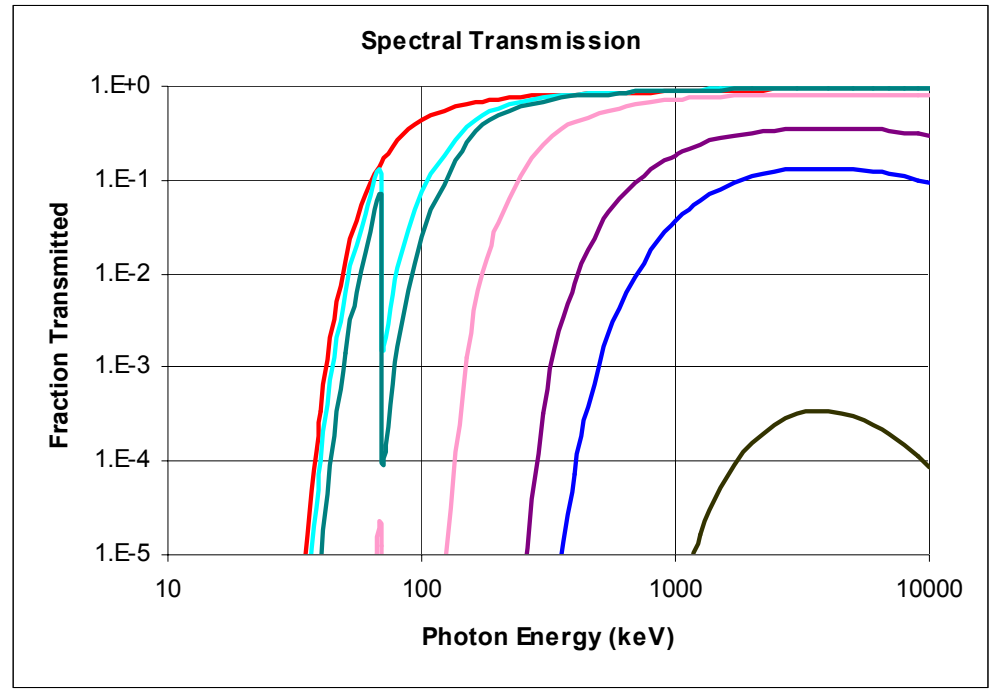

Fig. 6. Calculated x-ray spectral transmission after traversing blast shield $(1.4 \mathrm{~mm} \mathrm{SS})$ and absorbers used on radial view for shot 1177. Absorbers: $1.4 \mathrm{~mm}$ SS (red), remaining channels use W, $0.25 \mathrm{~mm}$ (light blue), $0.38 \mathrm{~mm}$ (green), $2.1 \mathrm{~mm}$ (pink), $12.5 \mathrm{~mm}$ (magenta), $25.4 \mathrm{~mm}$ (blue), $10.2 \mathrm{~mm}$ (black).

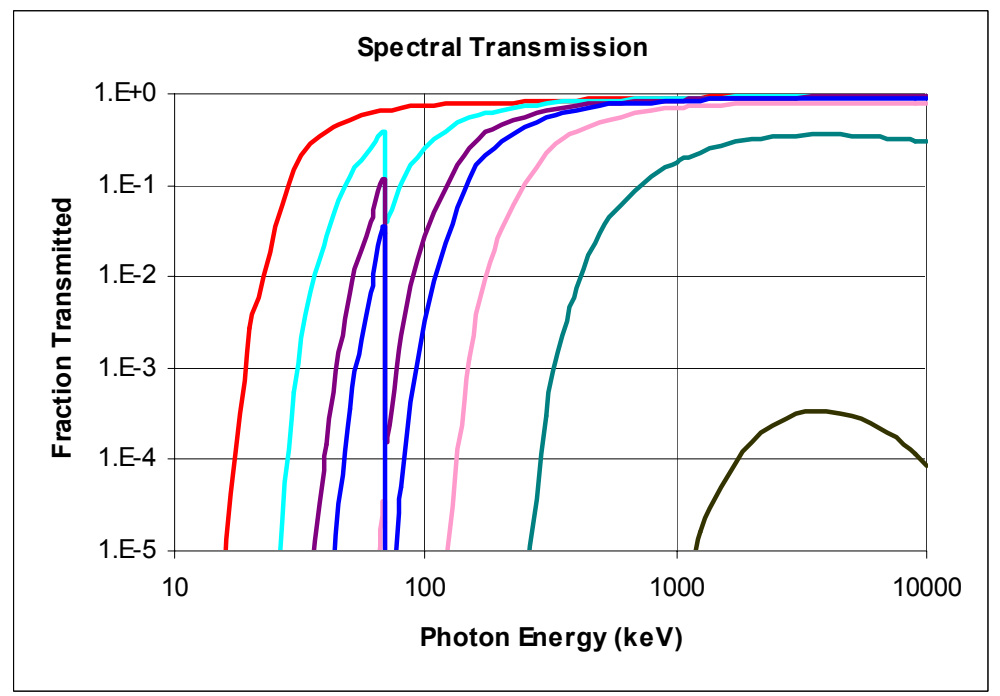

Fig. 7. Calculated $\mathrm{x}$-ray spectral transmission after traversing blast shield $(6.4 \mathrm{~mm} \mathrm{Al})$ and absorbers used on axial view for shot 1248. Absorbers: none (red), remaining channels use W, $0.13 \mathrm{~mm}$ (light blue), $0.38 \mathrm{~mm}$ (magenta), $0.64 \mathrm{~mm}$ (blue), $2.1 \mathrm{~mm}$ (pink), $12.7 \mathrm{~m}$ (green), $10.2 \mathrm{~mm}$ (black). 


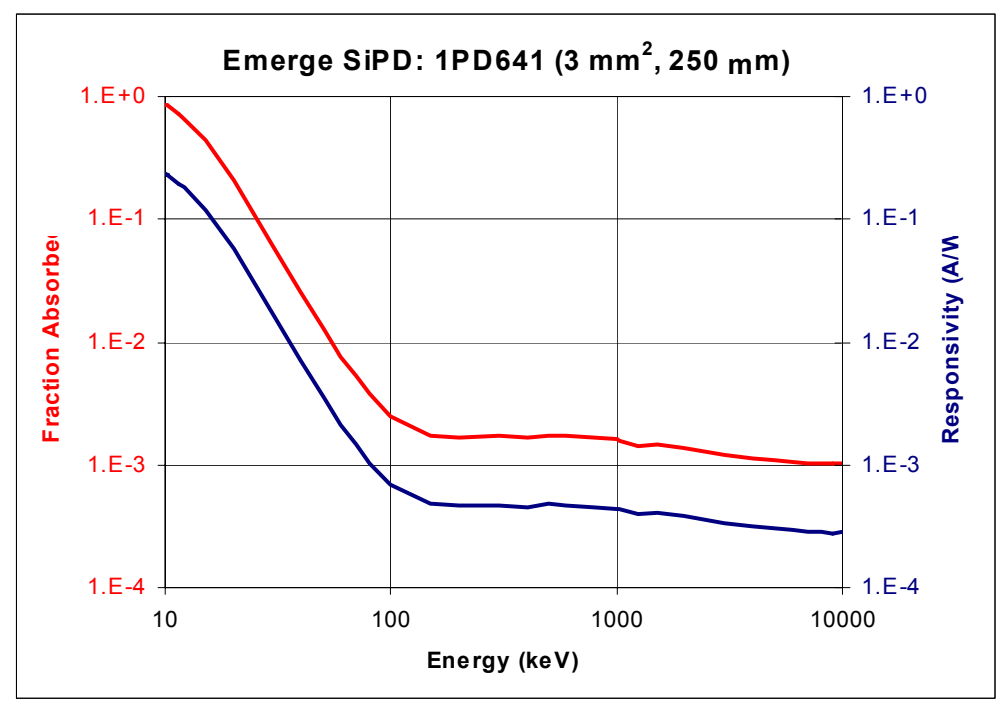

Fig. 8. Calculated spectral responsivity of Emerge Semiconductor Si PIN diodes used in HXRS. Si active area: $3 \mathrm{~mm}^{2}$, depletion depth: $250 \mu \mathrm{m}$.

Calibrated detector responses and absorber material characteristics are combined with measured data to unfold the spectral shape of the radiation source. This is described by the Fredholm integral equation of the first kind and its discretized representation:

$$
\begin{aligned}
& D_{j}(E, t)=\int_{E_{1}}^{E_{w}} S(E, t) R_{j}(E, t) d E+\varepsilon_{j} \quad j=1,2,3, \ldots 6 \\
& \mathrm{D}_{\mathrm{j}}(\mathrm{E}, \mathrm{t}) \approx \sum_{\mathrm{i}=1}^{\mathrm{n}} \mathrm{S}_{\mathrm{i}}(\mathrm{E}, \mathrm{t}) \mathrm{R}_{\mathrm{ij}}(\mathrm{E}, \mathrm{t})+\varepsilon_{\mathrm{j}} \quad \mathrm{j}=1,2,3, \ldots 6
\end{aligned}
$$

$\mathbf{R}_{\mathrm{ij}}(E, t)$ : Response of absorber + blast shield + PIN detector $\mathrm{j}$ over the discrete energy bins $\mathrm{i}=1$ to $\mathrm{n}$.

$\mathbf{S}_{\mathbf{i}}(E, t)$ : Time dependent $\mathrm{x}$-ray spectrum defined over the discrete energy bins $\mathrm{i}=1 \mathrm{to} \mathrm{n}$.

$\varepsilon_{\mathrm{j}}: \quad$ experimental uncertainties

\subsection{Unfold}

The iterative unfolding technique begins with a guess of the initial spectrum. Our $\mathrm{Z}$ shot data are taken from z-pinches of tungsten wire arrays (typically 300 wires, $10-\mathrm{mm}$ tall, $20-\mathrm{mm}$ diameter). The expected emission spectrum can be estimated to be a blackbody continuum emitter with a small fraction of energy in the characteristic $\mathrm{K}_{\alpha}$ lines at $\sim 60 \mathrm{keV}$, and/or high energy bremsstrahlung from electron-ion interactions in the Maxwellian plasma ${ }^{5}$. During the time evolution of the pinch, the spectral emission is probably a combination of the two effects. At stagnation, or peak of the pinch, we expect the non-thermal Maxwellian plasma condition to dominate and produce a bremsstrahlung spectrum. The trial spectrum is convolved with the known response matrix composed of detector response and absorbers. The calculated result is compared against the measurement for each detector. The trial spectrum is then modified to iterate the unfold process until the final result compares favorably with measurements to an acceptable uncertainty. If needed, the radiated power can be obtained from the calculated spectrum assuming a cylindrical Lambertian radiator.

$$
S(E, t)=A(t) e^{-E / k T(t)} \quad \text { (non-thermal) exponential bremstrahlung spectrum }
$$

$$
N(E)=N_{o} E^{2} e^{-E / k T} \quad \text { Maxwellian electron distribution }
$$




$$
\begin{aligned}
& S(E, t) \propto A(t) \frac{E^{3}}{e^{E / k T(t)}-1} \quad \text { Planckian spectrum } \\
& \mathrm{P}_{\text {tot }}(\mathrm{t})=\pi^{2} \mathrm{~A}_{\mathrm{S}} \int_{\mathrm{E}_{1}}^{\mathrm{E}_{2}} \mathrm{~S}(\mathrm{E}, \mathrm{t}) \mathrm{dE} \quad \text { cylindrical Lambertian emitter }
\end{aligned}
$$

\section{EXPERIMENTAL RESULTS}

The spectrometer has been fielded at the SNL Z facility to characterize the x-ray spectrum of wire array z-pinch experiments in both radial and axial views. Employing the differential filtering technique, materials like Al, $\mathrm{Fe}, \mathrm{W}$ ranging from $\mathrm{mm}$ to $\mathrm{cm}$ thicknesses are placed inside the collimator channels to characterize the spectral content ranging from $\sim 30-2000 \mathrm{keV}$. Due to blast conditions in $\mathrm{Z}$ experiments, a SS or Al debris shield placed in front of the collimation assembly also prefilters and hardens the incident $\mathrm{x}$-ray radiation.

Prior to installation in the seven channels, the Si PIN diodes were tested for relative sensitivity using $1.25 \mathrm{MeV}$ gammas from a Co-60 source. A set of seven diodes was selected with relative sensitivity of $\pm 5 \%$. The detectors were also "flat fielded" periodically either in open collimation mode or with the same set of absorbers for all channels on actual Z-shots, all illustrated in Fig. 9. The flat-fielded relative responses were used to normalize the data during postprocessing. All post-processing is done at the peak output time for each detector channel. Typically, the detector signals are accurate to $\pm 10 \%$. Occasionally, we have encountered greater measurement error due to unexpected issues with patch cables and attenuators.
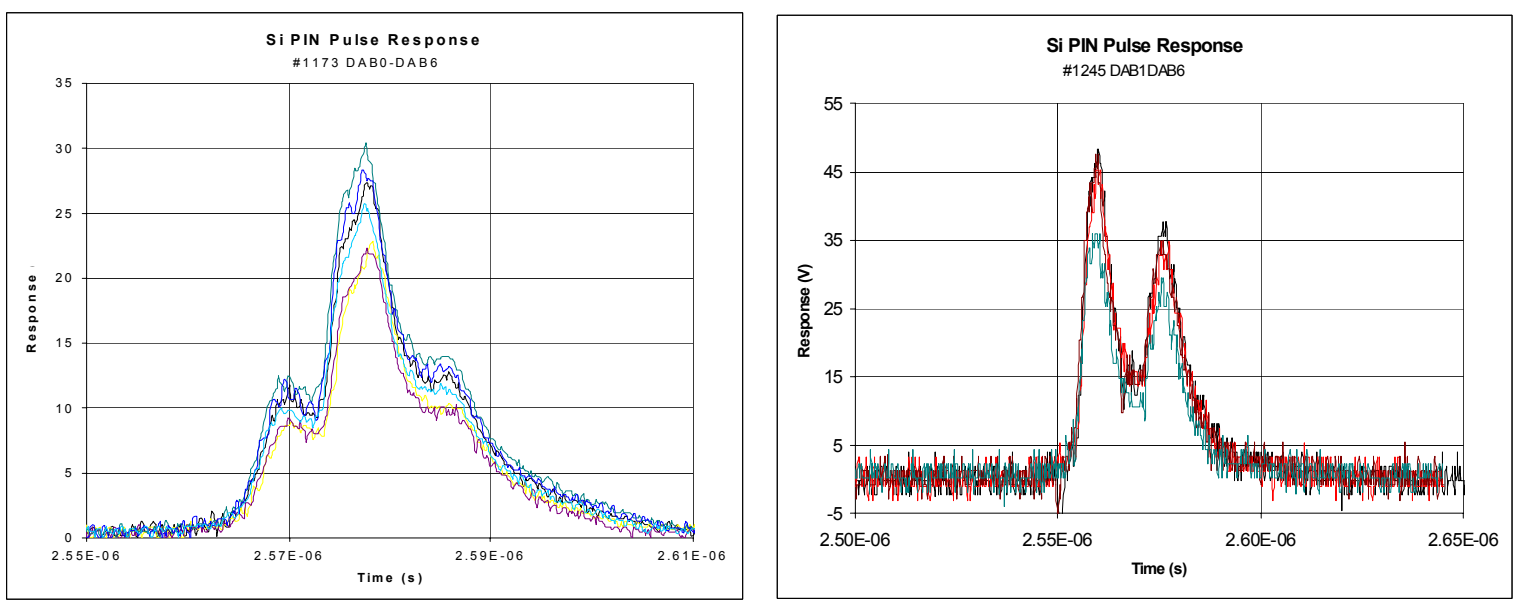

Fig.9. Detector Flat Fielding: a) open collimation on shot \#1173 for radial view. b) 0.5-mm Tungsten on shot \#1245 for axial view. 
Fig. 10 shows the seven-channel spectrometer radial data for shot \#1098, one of a series of radiation pulse shaping experiments investigating "light" array single hohlraums (20-mm dia. X 10-mm tall, $11.48 \mu \mathrm{m}$ dia. tungsten wires) to achieve high output power/temperature.
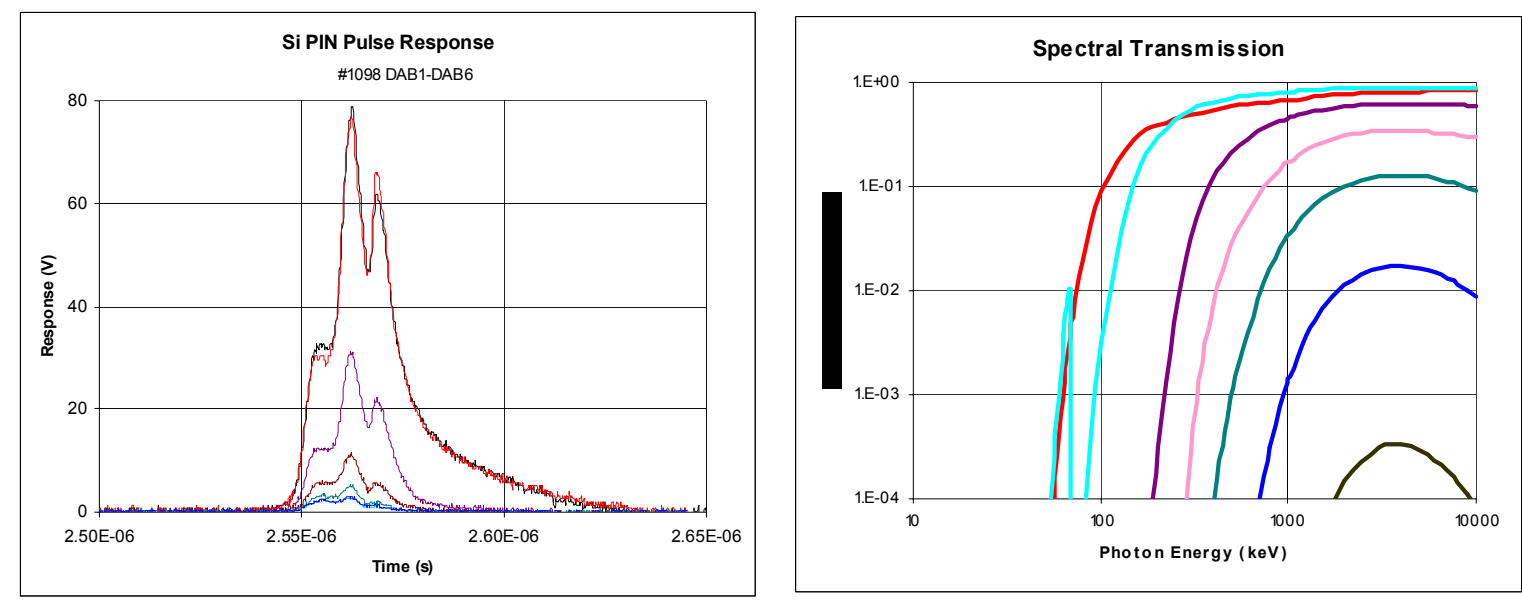

Fig. 10. a) Raw pulse responses for prototype spectrometer for radial view shot \#1098. b) Calculated spectral transmission of absorbers used.

The radial view data are found to agree well (Fig. 11) when the response functions (detector response shown in Fig. 8 and absorber transmission shown in Fig. 10b) are convolved with a decaying exponential bremsstrahlung spectrum produced by a $500-\mathrm{keV}$ to $1-\mathrm{MeV}$ Maxwellian electron distribution. The data for the highly absorbed channels (channels 5,6) show an apparent additonal higher temperature component.

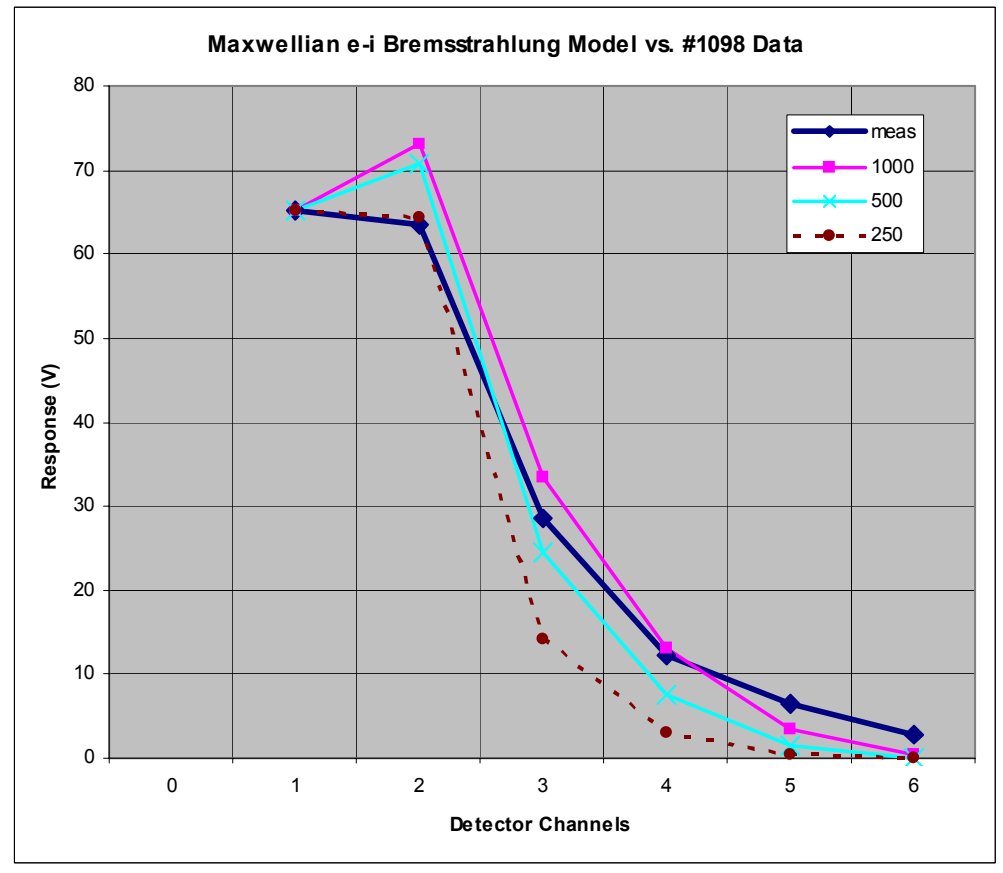

Fig. 11. Non-thermal exponential fit to peak output radial view data for shot \#1098. 
For the more refined compact spectrometer, seven channels were similary utilized in both the radial and axial view modes. In shot \#1177, one of a series of continuing pulse-shaping experiments using a single 300 -wire tungsten array (20-mm diameter, $10-\mathrm{mm}$ tall, $5 \mu \mathrm{m}$ diameter wires), we used a similar decaying exponential spectrum to convolve the response functions from Figs. 6 and 8. Figure 12 shows a nice fit at $1-1.5 \mathrm{MeV}$, a much higher temperature electron distribution. The additional higher temperature component indicated by channel 6 is also present.
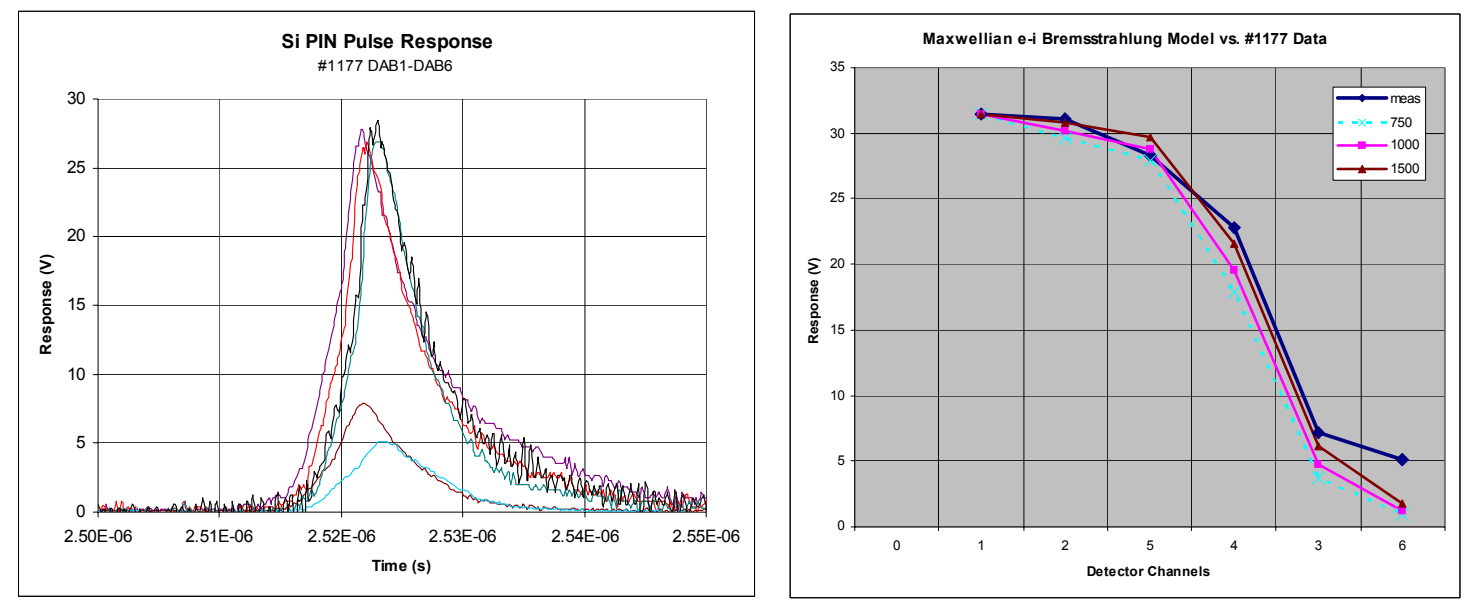

Fig. 12. a) Raw pulse responses for compact spectrometer for radial view shot \#1177. b) Non-thermal exponential fit to peak output data

With some modifications and mounting hardware, the spectrometer was fielded on axis to permit an axial view of the pinch. Data were taken for shot \#1248, part of a series of experiments investigating dynamic-hohlraum physics. These experiments used a double nested load composed of 14-mm-tall outer ( 240 wires, $40-\mathrm{mm}$ diameter, $5.24 \mu \mathrm{m}$ diameter wires) and inner (120 wires, 20 -mm diameter, $10.5 \mu \mathrm{m}$ diameter wires) cylindrical arrays. Again convolving a decaying exponential spectrum with the response functions from Fig. 7 and Fig. 8, we see a good fit for 500-750 keV electron temperature. These results as shown in Fig. 13 do not indicate an additional high temperature component.
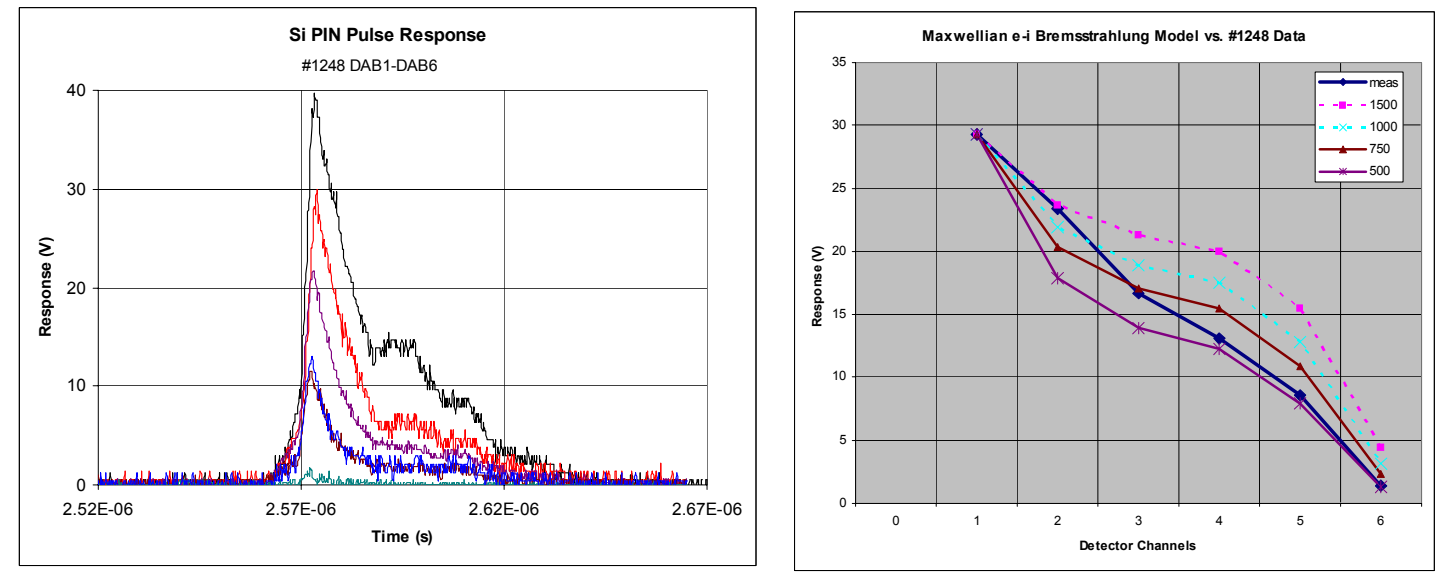

Fig. 13. a) Raw pulse responses for compact spectrometer for axial view shot \#1248. b) Non-thermal exponential fit to peak output data. 


\section{DISCUSSION}

The high-bandwidth measurement capability provided by this spectrometer is a powerful tool for characterizing timeevolved high-energy photon emission in a spatially-localized region. The data can be processed by suitable unfold codes and matrix solvers to yield a potential incident spectrum. Representative measurements and post-processing using a convolution method that folds a non-thermal exponential spectrum with calculated absorber transmission and detector response functions showed a nice fit at peak emission. To make maximum use of this instrument, a fully developed automated unfolding scheme is needed to extract the data over the complete time evolution. This code needs to be sufficiently flexible to permit a variety of input effects, such as the inclusion of line emission, $\mathrm{x}$-ray absorption due to plasma opacity, additional high-energy components due to accelerated electrons, etc.

We are continuing to develop this methodology and plan to implement the kernel of UFO, UNSPEC, or YOGI within IDL to suit our requirements. As we improve in applying and developing mathematical unfolds, spectral data obtained via the HXRS, varied over a spread of angles ranging from radial to axial views, will help us to further understand the pinch physics at $Z$ and the soon-to-be-completed ZR. For lower energy spectral characterization, this instrument can be easily changed to a Ross-pair configuration using appropriate filters and absorbers to provide a direct sampling of the $\mathrm{x}$-ray spectrum in the mid-energy range from a few to $\sim 115 \mathrm{keV}$.

\section{ACKNOWLEDGMENTS}

We would like to thank Carlos Ruiz, Greg Rochau, Gordon Chandler, and especially Dave Fehl for stimulating discussions and assistance with spectral unfold techniques. The measurements at $Z$ could not have been done without the tireless dedication and efforts provided by Chuck Dickerson, Jerry Taylor, and the Z operations staff. The NSTec STL staff of Guy Leach, Glenn Anthony, and Wayne Lenhard provided critical support with mechanical design and machining. Finally, we thank Ron Head (NSTec LV) for help in characterizing the absolute response of the Si PIN detectors and the linac operations staff at the Idaho Accelerator Center for assistance with the temporal response measurements.

\section{REFERENCES}

1. $\quad$ UNSPEC (Unfolding X-Ray Spectrum), AEN ESTS0827

2. UFO (Unfold Operator), Sandia Report Sand82-0396, UC-705, 1991

3. D. J. Johnson, J. Appl. Phys. 45, 1147 (1974)

4. $\quad$ I. V. Khutoretsky, Rev. Sci. Instrum. 66 (1), 773 (1995)

5. G. H. McCall, J. Phys. D: Appl. Phys., 15, 823-831 (1982)

This manuscript has been authored by Bechtel Nevada and National Security Technologies, LLC under Contract Numbers DE-AC08-96NV11718 and DE-AC52-06NV25946, respectively, with the U.S. Department of Energy. The United States Government retains and the publisher, by accepting the article for publication, acknowledges that the United States Government retains a non-exclusive, paid-up, irrevocable, world-wide license to publish or reproduce the published form of this manuscript, or allow others to do so, for United States Government purposes. 